\title{
Educomunicación, webradio y educación no formal en un contexto Erasmus+: la experiencia de Europa on air
}

\section{(Educommunication, Web radio and Non-Formal Education in an Erasmus + Context: the Europa On Air Experience)}

\author{
Miguel Ángel Ortiz Sobrino \\ Universidad Complutense de Madrid (España) \\ Nereida López Vidales \\ Universidad de Valladolid (España) \\ Juan Carlos González Pérez \\ Universidad Complutense de Madrid (España)
}

DOI: https://doi.org/10.5944/ried.25.1.30641

\section{Cómo referenciar este artículo:}

Ortiz Sobrino, M. A., López Vidales, N., y González Pérez, J. C. (2022). Educomunicación, webradio y educación no formal en un contexto Erasmus+: la experiencia de Europa on air. RIED. Revista Iberoamericana de Educación a Distancia, 25(1), pp. 163-181. https://doi.org/10.5944/ried.25.1.30641

\section{Resumen}

La webradio y las redes sociales se han revelado como herramientas esenciales para el aprendizaje y la adquisición de competencias en comunicación. También algunas acciones del programa Erasmus + han devenido en escenario idóneo para la educación no formal. Este artículo presenta la experiencia de Europa on air, un proyecto Erasmus+ en el que los alumnos participantes adquirieron competencias de comunicación a través de la webradio y de las redes sociales, así como otras de carácter lingüístico, intercultural y relacionadas con la ciudadanía europea y el respeto a la diversidad. Como principales resultados de la experiencia destacan su capacitación en técnicas de producción y emisión de programas radiofónicos y el uso de las redes sociales como vehículos de comunicación para difundir la diversidad cultural y social de la Unión Europea (UE). Igualmente, el proyecto sirvió para que mejoraran sus competencias idiomáticas en lengua francesa e inglesa. Paralelamente, el concepto de inclusión y ciudadanía europea se promovió a través de la convivencia con familias de estudiantes e instituciones educativas de cada país participante. Todas las competencias adquiridas fueron oficializadas a través de Europass. Europa on air se basó en estancias semanales en los cinco países 
acogidos al proyecto en las que los alumnos asistieron a actividades culturales, deportivas y sociales que sirvieron de base para elaborar los contenidos de los programas que realizaron para la webradio de cada institución educativa, que después fueron viralizados en las redes sociales. Las conclusiones generales completan otras aportaciones de la literatura científica y experiencias en la radio pública.

Palabras clave: webradio; redes sociales; Erasmus+; competencias educativas; educomunicación; Educación no formal.

\begin{abstract}
Web radio and social media have proved to be essential tools for the acquisition of skills and learning. Some actions of the Erasmus+ program have also become an ideal scenario for non-formal education. This article presents the experience of Europa on air, an Erasmus+ project in which the participating students have acquired communication skills through web radio and social networks, as well as other linguistic, intercultural and European citizenshiprelated skills and respect for diversity. The main results of the experience include training in radio program production and broadcasting techniques and the use of social networks as a communication vehicle to disseminate the cultural and social diversity of the European Union (EU). The project also helped them to improve their language skills in French and English. At the same time, the concept of inclusion and European citizenship was promoted through living with students' families and educational institutions in each country. All the competencies acquired were officialized through Europass. Europa on air was based on weekly stays in the five countries involved in the project, during which the students attended cultural, sports and social activities that served as the basis for preparing the contents of the programs they made for the webradio of each educational institution, which were then viralized on social networks. The general conclusions complement other contributions from the existing scientific literature and experiences in public radio.
\end{abstract}

Keywords: web radio; social media; Erasmus+; educational competencies; educommunication; non-formal education.

La movilidad de estudiantes ha sido uno de los principales ámbitos de acción política en el Espacio Europeo de Educación Superior (EEES), ya que mejora el desarrollo de competencias, fomenta la cooperación y promueve el respeto a la diversidad y el plurilingüismo, que sustentan la diversidad pluricultural europea (Tur et al., 2019). En ese espacio de aprendizaje la comunicación en general, y la webradio y las redes sociales en particular, han adquirido un protagonismo notable en el marco de la educación no formal, como ha sido puesto de manifiesto por la literatura científica (Rendón López y Ortega Carrillo, 2015; Celaya et al., 2020).

Autores como Blas García (2020) han reparado en el binomio educacióncomunicación y en la educomunicación como dimensión teórico-práctica para la adquisición de competencias, gestión del conocimiento y producción de contenidos 
educativos. Sostiene este educomunicador que la formación en y con medios de comunicación nos lleva a poder hacer efectiva la necesidad de una gestión democrática del conocimiento, una de las líneas básicas del modelo educativo de los próximos años. Su planteamiento teórico sitúa el foco en que la importancia de la comunicación es esencial en el aprendizaje para concluir que, probablemente, el éxito de la educación radica hoy en una buena utilización de las técnicas y herramientas de comunicación en la relación alumno/profesor.

Algunos investigadores se han referido al sentido polisémico de la educomunicación, aunque alguno de ellos lo identifican como una adquisición de competencias comunicativas o una alfabetización mediática para estudiantes y profesores. (Chiappe Laverde y Arias Vallejo, 2016; Rodríguez García, 2017; Fuentes Cancell et al., 2021). Por ello, no es casual el interés desde la universidad y desde la escuela por incorporar los medios de comunicación al proceso de aprendizaje reglado o en la educación no formal.

A raíz de la generalización del uso de las TIC entre los alumnos y profesores, ciertos soportes de comunicación vinculados a internet y las nuevas plataformas de difusión se han incorporado como herramientas para la educomunicación y el aprendizaje. Ese es el caso de la webradio, los podcasts y los social media, vinculados directamente a la web 2.0 (Vázquez, 2007; López Vidales y González Aldea, 2014; García Marín, 2020). Este entorno de la web 2.o ha venido a fomentar las capacidades de aprendizaje, comunicación y producción de contenidos de los profesores y de los estudiantes, dando lugar a un nuevo paradigma educativo en la última década (Durán Becerra y Tejedor Calvo, 2017). Precisamente este escenario de convergencia entre webradio y las redes sociales en favor de la educación ha sido aprovechado por el Programa Erasmus+ en los últimos años.

En ese contexto de aprendizaje propiciado por el entorno Erasmus, se implementó, entre 2017 y 2020, una acción especial K2 denominada Europa on air en el que participaron instituciones educativas de Francia, Italia, República Checa, Reino Unido y España. El proyecto fue una iniciativa encaminada a promover la educación para la ciudadanía europea, la alfabetización mediática y la adquisición de competencias interculturales que han sido, en las dos últimas décadas, algunos de los objetivos del programa Erasmus+ promovido y financiado por la Unión Europea (Aguaded y Martín Pena, 2013; Adalid Donat y Carmona Rodríguez, 2015; Asenjo Gómez, 2017).

\section{ERASMUS+ COMO ESCENARIO PARA LA EDUCACIÓN, LA ALFABETIZACIÓN MEDIÁTICA Y LAS COMPETENCIAS INTERCULTURALES}

Erasmus+ es el programa integrado en Unión Europea (UE) para la Educación, Formación, Juventud y Deporte. El proyecto original comenzó en 1987 como un programa de intercambio que ofrecía a estudiantes universitarios la posibilidad de 
aprender y enriquecerse estudiando en el extranjero. Desde sus inicios, se convirtió en una herramienta de intercambio cultural y académico esencial para el aprendizaje.

Las dos principales acciones relacionadas con juventud e innovación, especialmente enfocada en instituciones educativas, son las acciones KA1 y KA2. Las KA1 fomentan la movilidad de estudiantes, trabajadores, voluntarios, animadores juveniles y jóvenes. A través de ella, las organizaciones e instituciones educativas pueden enviar estudiantes y colaboradores a países participantes, acoger estudiantes y personas de otros europeos, así como organizar actividades de docencia, formación, aprendizaje y voluntariado. Por su parte, las KA2 están pensadas para el desarrollo de los sectores de la educación, la formación y la juventud mediante cinco actividades principales: asociaciones estratégicas, alianzas para el conocimiento, alianzas para las competencias sectoriales, desarrollo de las capacidades en el ámbito de la educación superior y proyectos de desarrollo de las capacidades entre los jóvenes ${ }^{1}$.

\section{Competencias y cualificaciones para un contexto europeo de educación}

El Espacio Europeo de Educación para 2025, diseñado desde la Comisión Europea, considera la educación y la cultura los motores para generar empleo y crecimiento económico (Asenjo y Asenjo Gómez, 2020). Para facilitar la movilidad educativa y la búsqueda de empleo por los países, la Unión Europea ha creado una serie de documentos que oficializan las titulaciones, competencias y cualificaciones profesionales o académicas de los estudiantes participantes en alguno de los proyectos incluidos en la filosofía de Erasmus+ en todo el territorio europeo ${ }^{2}$. El instrumento que responde a esta iniciativa es el modelo Europass que ha sido diseñado desde cuatro vertientes claramente diferenciadas:

1. Europass para la educación y la formación

2. Europass para el empleo y la contratación

3. Europass para la orientación profesional

4. Marco Europeo de Cualificaciones (MEC)

Tras la participación en un proyecto Erasmus+, las propias instituciones educativas inmersas en los proyectos pueden expedir cualificaciones digitales y otras certificaciones de aprendizaje gracias al marco de credenciales digitales de Europass. Esta herramienta proporciona a los alumnos, a las empresas, a los centros educativos y al resto de organismos autorizados, un procedimiento fiable para verificar la autenticidad de dichas credenciales. 


\section{EUROPA ON AIR: COMPETENCIAS INTERCULTURALES, EDUCACIÓN PARA LA CIUDADANÍA EUROPEA Y CAPACITACIÓN EN COMUNICACIÓN A TRAVÉS DE LA WEBRADIO Y LOS SOCIAL MEDIA}

El proyecto Europa on air fue una acción de educación no formal KA2/Clave1, en el que participaron cuatro instituciones educativas y una organización sin ánimo de lucro, pertenecientes a cinco países europeos. El proyecto fue coordinado por el Lycée Gustave Eiffel, de Burdeos (Francia), en el que participaron como socios colaboradores el Liceo Classico Francesco de Sanctis, de Salerno (Italia), Radioexpert y la Universidad de Brno (República Checa), RedTower Ltd. de Londres (Reino Unido) y la Universidad Complutense de Madrid (España).

\section{Figura 1}

Logo oficial del proyecto Europa on air
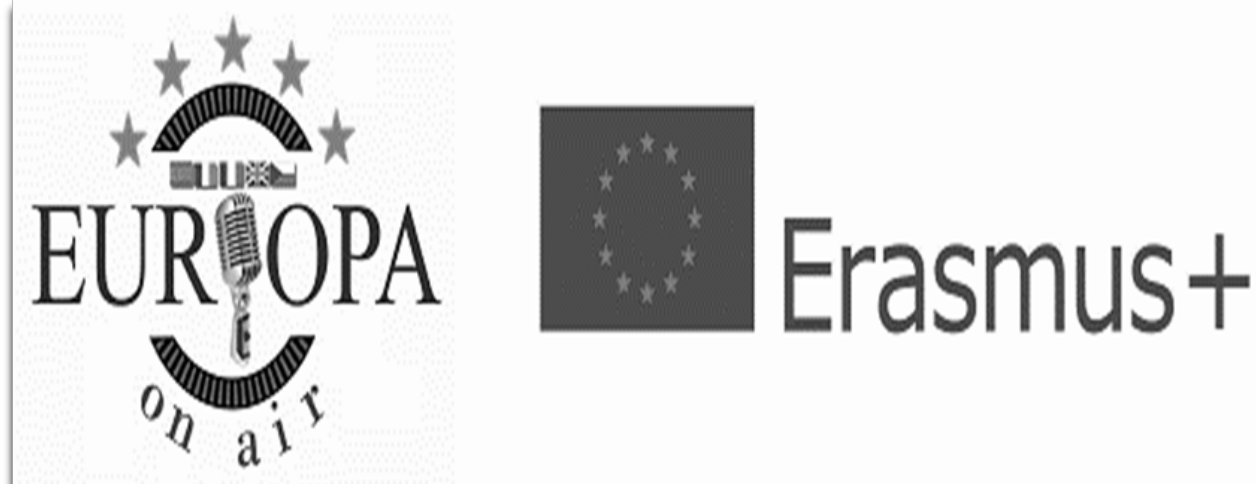

Fuente: https://sites.google.com/site/europaonair/

El Lycée Gustave Eiffel de Burdeos es un centro educativo politécnico que imparte tanto formación reglada (bachiller), como diferentes grados de formación y cualificación profesional. El Liceo Classico Francesco De Sanctis es un instituto de enseñanza media de la ciudad de Salerno. Radioexpert de Brno es una emisora universitaria asociada a la universidad pública de aquella ciudad checa. Por su parte, Red Tower es un proyecto fundacional del Reino Unido que se centra en la interculturalidad de jóvenes británicos de diferentes razas, etnias y religiones. Finalmente, la Universidad Complutense de Madrid (España) es una institución de educación superior radicada en Madrid que acoge a cerca de ochenta mil alumnos, que cursan alguno de sus Grados, Doctorados y títulos propios.

En el proyecto, financiado por la Unión Europea, estuvieron implicados ciento cincuenta alumnos y diez profesores de las cinco instituciones promotoras del 
proyecto. Se trataba de estudiantes de diferentes nacionalidades y edades, con distintos niveles de formación (estudiantes de bachillerato, grado superior de formación profesional y universitarios) y diversos orígenes sociales. La participación por nacionalidades no fue homogénea, como puede observarse en el siguiente gráfico (2). Francia e Italia aportaron, respectivamente, cincuenta y veinticinco estudiantes, mientras que el resto de los países incorporaron al proyecto veinticinco estudiantes cada uno de ellos.

\section{Figura 2}

Participantes por nacionalidades en Europa on air

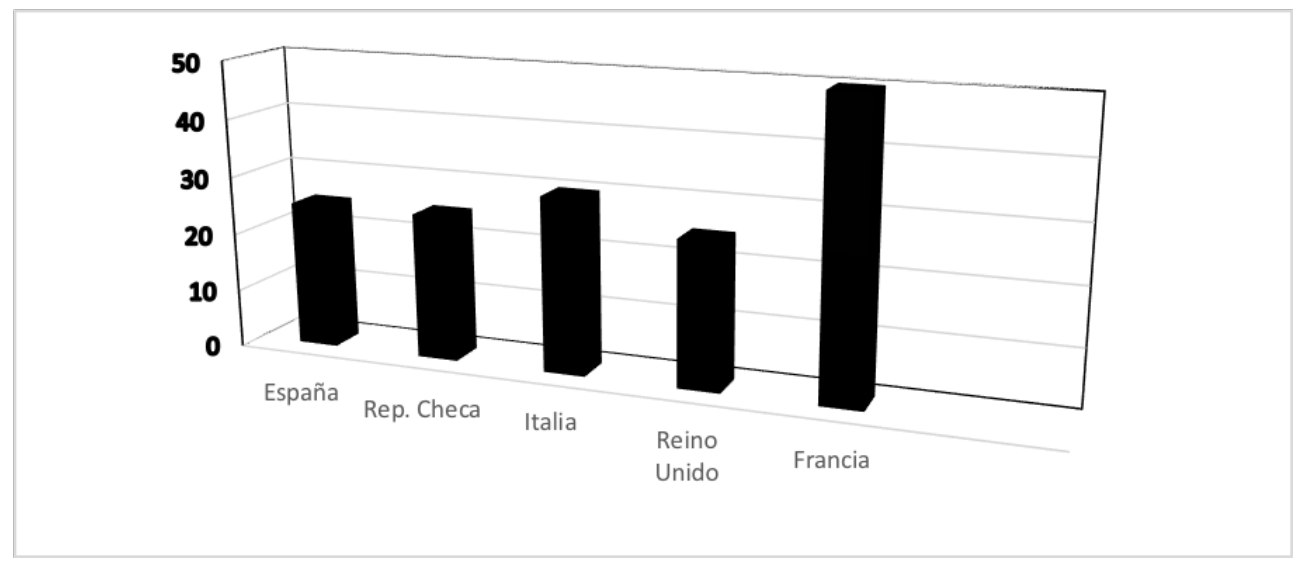

Fuente: Elaboración propia

A través de este proyecto, las instituciones promotoras, comprometidas con la idea de una Europa de los ciudadanos y el potencial de los medios de comunicación en la educación de los adolescentes y jóvenes, promovieron la movilidad de jóvenes estudiantes y profesores para conocer la cultura y realidad político-social de cada uno de los países participantes. Entre las actividades programadas, se contemplaba una semana en cada uno de los países con el objetivo de conocer las instituciones y la cultura del país de acogida. Las experiencias de esa estancia fueron puestas en antena en un programa de radio que se emitió vía streaming en la emisora online de cada uno de los centros educativos en sus respectivos países. Además, el proyecto contaba con una identidad en redes sociales y una página web general y otra específica de cada país, donde se informaba de las actividades del proyecto ${ }^{3}$.

Los idiomas utilizados para las emisiones en todos los países fueron la lengua inglesa y la francesa, aunque durante la movilidad por los distintos países pudieron familiarizarse con su lengua (italiano, español o checo) durante la convivencia con las familias de acogida o en las actividades de ocio fuera del programa. 


\section{OBJETIVOS DEL PROYECTO EUROPA ON AIR}

El proyecto Europa on air tenía como objetivos principales la promoción de concepto de ciudadanía europea, la integración de los jóvenes europeos en la diversidad cultural, social e idiomática de la Unión Europea, el respeto a la diferencia, así como el conocimiento de la webradio y las redes sociales como vehículos de comunicación y aprendizaje. Como objetivos específicos del proyecto figuraban los siguientes:

a. La convivencia y el conocimiento de la realidad social de cada uno de los países integrantes del proyecto.

b. Promover entre los jóvenes la idea de una Europa de los ciudadanos.

c. Conocimiento de la realidad cultural a través de diferentes actividades programadas por la organización del proyecto en cada uno de los países.

d. Difusión de la realidad multicultural, étnica e idiomática mediante la convivencia de los alumnos de los diferentes países.

e. Potenciar la capacidad de comunicación entre los jóvenes europeos y la adquisición de competencias para el uso de las redes sociales y la webradio como herramientas de comunicación, de difusión cultural y de vertebración de la multiculturalidad en la Unión Europea.

f. Crear una red de relaciones europea entre los estudiantes que participaban en el proyecto para futuras acciones y convivencias en común que promovieran la conciencia de ciudanía europea.

\section{METODOLOGÍAS Y PLANTEAMIENTO DEL PROYECTO}

Europa on air se basó en estancias semanales de los estudiantes por los cinco países implicados en el proyecto durante las cuales convivían con las familias de los alumnos del país para promover el conocimiento de su realidad sociocultural, para mejorar sus competencias idiomáticas en lengua inglesa y francesa, así como un acercamiento a otras lenguas de alguno de los países de acogida: Italia, República Checa y España.

Durante las estancias, los alumnos asistieron a actividades culturales, tales como visitas a museos, arquitectura del país, encuentros con personalidades locales de la política, la cultura o el deporte, y otras de convivencia desarrolladas en los diferentes centros educativos participantes. Esta experiencia cultural y los encuentros con los protagonistas de la cultura local sirvió como base de los contenidos de un programa magazín que los alumnos realizaron en los estudios de radio de cada institución educativa y quedaron alojados en el repositorio de las webs corporativas de cada uno de los partners promotores y otras plataformas de difusión digital, como iVoox en el caso español. También se produjeron programas en sistema de conexión múltiple entre los participantes desde sus respectivos países a través del medio radiofónico 
(webradio) para poner en valor a la radio como medio de unificación, potenciación e interculturalidad entre países europeos. Estos contenidos eran viralizados por los alumnos a través de Facebook e Instagram.

Con el fin de evaluar el impacto de la movilidad de los estudiantes y valorar si las actividades programadas estaban contribuyendo a los objetivos planteados en el proyecto Europa on air, se diseñaron dos cuestionarios: uno, contestado por los estudiantes a su llegada a cada país, una vez que se iniciaron las actividades programadas en la ciudad de acogida; otro, completado por los estudiantes al finalizar su estancia en esa ciudad. A través de ambos cuestionarios se pretendió valorar si los estudiantes habían reforzado su conocimiento del país que habían visitado, y si la imagen que tenían de ese lugar y sus ciudadanos a su llegada había cambiado. Para evaluar la consecución de objetivos del proyecto, se entregaba a cada uno de los alumnos un cuestionario de llegada en cada estancia con el fin de determinar su grado de conocimiento de la cultura, el idioma y los aspectos sociales del país de acogida. Al finalizar la movilidad, el alumno cumplimentaba un cuestionario de salida para valorar el grado de conocimiento y competencias adquiridas.

El cuestionario de entrada constaba de once preguntas con opción múltiple y una final abierta. Las diez preguntas cerradas permitían determinar el país de procedencia del alumno, el grado de conocimiento del idioma y la cultura del país de acogida de cada movilidad Erasmus, así como comparar la cultura y sociedad del país de acogida en relación con el suyo. Igualmente, se daba al alumno la oportunidad de valorar la movilidad Erasmus como instrumento para fomentar la relación entre los alumnos europeos y para eliminar las barreras culturales o de convivencia entre los jóvenes de los diferentes países de la Unión Europea. La pregunta abierta final ofrecía al alumno la posibilidad de aportar ideas para mejorar el conocimiento de la Unión Europea y en especial relacionadas con el uso de la radio y las escuelas para fortalecer los valores europeos entre los jóvenes y adolescentes.

El cuestionario de salida estaba encaminado a, por un lado, observar si la idea preconcebida a la llegada de un país se alteraba después de la estancia y, por otro, si valoraban que la webradio y la movilidad contribuía a fortalecer los valores europeos entre los jóvenes estudiantes. A diferencia del primer cuestionario, en el segundo se ofrecían dos preguntas abiertas: en la primera se pedía al estudiante que detallara las razones por las cuales la movilidad y particularmente la webradio permitían fortalecer los valores y competencias culturales e idiomáticas; en la segunda, se solicitaba al alumno la coherencia del plan de actividades del proyecto con sus objetivos.

Las estancias y la cumplimentación de los cuestionarios tuvieron lugar en las siguientes fechas: Salerno (Italia), del 2 al 7 de octubre de 2017 y del 20 al 25 de junio de 2019; Burdeos, del 19 al 23 de abril de 2018; Brno (República Checa), del 29 de abril al 4 de mayo de 2019 y Madrid (España) del 1 al 6 de octubre de 2018. En la reunión transnacional celebrada en Londres no se aplicaron los cuestionarios por tratarse de una actividad de puesta en común y planificación. 


\section{La webradio y los social media como herramientas vertebradoras del proyecto Europa on air}

Las relaciones interpersonales y la comunicación fueron recursos fundamentales que sirvieron de columna vertebral para la convivencia y la integración de los estudiantes y profesores en el proyecto. Las relaciones personales se propiciaron tanto en el entorno familiar, como en la participación de las actividades. Paralelamente, la adquisición de competencias y la alfabetización mediática fueron promovidas mediante la webradio escolar y universitaria y el uso de los social media.

Las radios escolares y universitarias se emplean desde hace décadas en diferentes países como instrumento de comunicación y alfabetización de los estudiantes (MartínGracia et al., 2018; Pérez-Femenía e Iglesias-García, 2020), sobre todo después de la aparición del streaming. Como han puesto de manifiesto algunos autores, en los últimos años se han incrementado los estudios sobre la radio en contextos universitarios como una herramienta capaz de mejorar la competencia mediática de los alumnos universitarios (Ortiz-Sobrino et al., 2020). Estas emisoras son una realidad en muchos países de la Unión Europea: Italia, España y Francia, entre otros (Ortiz Sobrino et al., 2014). Por su parte, las redes sociales se han convertido en auténticos vehículos de comunicación entre los jóvenes y adolescentes, no solo como instrumentos de relación interpersonal, sino como medio para viralizar sus mensajes.

\section{RESULTADOS: EDUCOMUNICACIÓN, INTERCULTURALIDAD, COMPETENCIA LINGÜÍSTICA, CIUDADANÍA EUROPEA Y DIVERSIDAD}

De las respuestas recogidas en los cuestionarios de entrada y salida de las estancias programadas durante el proyecto Europa on air, se extraen los resultados que se exponen a continuación.

El 100\% de los cuestionarios cumplimentados por los estudiantes revelan que las diferentes movilidades organizadas les han permitido conocer mejor cada uno de los territorios de la Unión Europea visitados, su cultura y su realidad social. De igual manera, todos los alumnos han tomado conciencia de que la movilidad Erasmus contribuye a la supresión de las barreras culturales y sociales entre las diferentes nacionalidades, lo que permite avanzar en el concepto de ciudadanía europea. Aunque fueron conscientes de la singularidad de cada país, han podido comprobar que cada una de las regiones visitadas estaban comprometidas con la ciudadanía europea. Finalmente, la mayoría de los alumnos que han participado en Europa on air, señalaban, de interés notable, la búsqueda de espacios de convivencia común entre jóvenes de diferentes países.

Como ya se puso de manifiesto en movilidades anteriores, existe una opinión unánime al considerar este tipo de movilidad como un instrumento para lograr una 
mayor conciencia europea entre sus jóvenes. Todos los estudiantes creen que este tipo de experiencias apoyan la integración de los jóvenes en un proyecto europeo común; Europa on air es una oportunidad para potenciar la unidad y las relaciones entre los jóvenes europeos.

Al inicio de la movilidad, el grupo de estudiantes, a excepción de uno, se interesó por los siguientes temas:

a. Eliminar barreras culturales y de convivencia

b. Construir una ciudadanía europea con valores comunes

c. Buscar espacios de convivencia entre jóvenes de países diferentes

d. Conocer el sistema educativo del país y compararlo con el mío

$\mathrm{Al}$ final de la experiencia Erasmus+, el total de los estudiantes mantuvieron ese nivel de interés por las mismas cuestiones, aunque incorporaron un tema nuevo: conocer a sus vecinos europeos.

En relación con los asuntos europeos, el 100\% de los participantes manifestaron que, al término del proyecto, disponían de un mayor conocimiento e interés por las acciones europeas encaminadas a la integración, la convivencia y la ruptura de las barreras socio culturales entre los diferentes países.

A continuación, se detallan los resultados de la experiencia en relación a la educomunicación, la competencia lingüística, la ciudadanía europea así como su diversidad e interculturalidad.

\section{Europa on air en la webradio y las redes sociales: un ejercicio de alfabetización mediática y competencia lingüística}

La webradio y las redes sociales actuaron como columna vertebral de la convivencia y la integración entre los participantes en este proyecto Erasmus+. La producción de una serie de programas emitidos en streaming a través de las webradios de las instituciones participantes, fue uno de los elementos vertebradores del proyecto. Con este fin, la web de cada una de las instituciones educativas colaboradoras se convirtió en un espacio de trabajo donde los estudiantes pudieron crear un podcast que reflejó la experiencia vivida durante esos días.

Simultáneamente, se creó un perfil específico de Facebook para el proyecto donde se actualizaron galerías de imágenes y actividades realizadas en cada una de las movilidades donde participaron los estudiantes, además de la publicación de artículos sobre los partners y otros de interés del proyecto ${ }^{4}$. Además, se creó un canal de Instagram que contó con perfiles específicos en cada movilidad, mediante los cuales se desarrollaron juegos interactivos y acciones con grupos de trabajo (https://www.instagram.com/europaonair/). En el caso español, se creó una cuenta específica en Facebook a través de la que se informó de los viajes, acciones realizadas y aprendizaje obtenido en las movilidades (https://www.facebook.com/ 
EuropaonAirSpain/). Las redes sociales elegidas representaron al público objetivo medio que participó en el proyecto y al que se destinaron las acciones: jóvenes y docentes.

El objetivo de esta actividad estaba centrado en dar a los estudiantes la posibilidad de elaborar materiales para trabajar eficazmente el uso de las tecnologías de la comunicación y la información, especialmente en el ámbito radiofónico y de los social media, de tal manera que su elaboración permitiera complementar la estrategia de difusión de las actividades realizadas y la adquisición de competencias y destrezas para la comunicación a través de la radio, las redes sociales y la web.

Para elaborar tanto los contenidos de los programas que se emitían a través de la webradio, como para la viralización de mensajes y contenidos mediante las redes sociales, los alumnos tuvieron que adquirir competencias que les permitieran abordar la fase de diseño, producción y emisión digital de los contenidos, En el caso de la radio, los estudiantes se capacitaron previamente en técnicas de producción y guion radiofónico, locución y dominio de la tecnología aplicada a la realización y difusión de programas en streaming. Para suministrar contenidos de imagen y video que acompañaran a las publicaciones web, los alumnos se capacitaron en técnicas de reporterismo fotográfico, grabación y edición digital de reportajes y entrevistas en video. De igual manera, adquirieron el dominio del lenguaje propio de los textos destinados a los social media que completaron las competencias orales sobre las que empezaron a trabajar desde sus primeros años escolares y que han sido abordadas desde la literatura científica (Fallarino et al., 2020). De forma transversal, esta experiencia de convivencia y capacitación potenció la capacidad de trabajo en equipo y la gestión del tiempo entre los estudiantes.

La creación de este medio de comunicación colaborativo implicó para el 100\% de los estudiantes la adquisición de habilidades importantes tanto para los estudiantes, como para los tutores involucrados: dominio de la técnica de comunicación radiofónica, trabajo en equipo y capacidad de producción de contenidos para la radio. La experiencia supuso, igualmente, la capacitación de los estudiantes para el trabajo colaborativo (González Conde et al., 2018; Rendón López y Ortega Carrillo, 2015).

En este sentido, todas las respuestas a las preguntas abiertas del cuestionario incidían en la necesidad de aprovechar la webradio para difundir los valores europeos entre los escolares y estudiantes universitarios. Los jóvenes participantes planteaban, por ejemplo, emitir contenidos europeos para la radio pública de todos los países, programar en este tipo de emisoras música producida por la juventud o invitar a jóvenes y adolescentes europeos a participar en las emisiones desde la webradio. Al final del proyecto, la propuesta sugerida más aplicable al proyecto Europa on air se centraba en ampliar las actividades de la webradio no solo en el contexto Erasmus+, sino también en las actividades cotidianas de cada centro educativo, como una competencia más en el aprendizaje. 


\section{La Web 2.o como herramienta estratégica}

La estrategia general de comunicación de Europa on air se articuló sobre el desarrollo de una página web específica del proyecto, en francés, con toda la información sobre su planificación y su desarrollo: puntos clave del proyecto, miembros participantes, actividades realizadas, audios, imágenes, videos y noticias publicadas. En esta web se alojaron los programas elaborados durante cada movilidad y los programas multiplex realizados en conexión directa y simultánea entre todas las radios de los centros educativos. El idioma inglés fue la lengua utilizada en la producción y emisión de todos los programas de radio. En las redes sociales se incluyeron galerías de imágenes, vídeos y resúmenes específicos de las movilidades que aparecen en la página web oficial ${ }^{5}$. Todos los alumnos participaron en este tipo de tareas, lo que implicó la adquisición de competencias para el dominio y conocimiento de la web 2.o como herramienta para la comunicación y el aprendizaje.

Cada país desarrolló su web específica. En el caso español se utilizó la web institucional de la emisora de la Universidad Complutense. En España, el programa se emitió en InfoRadio ${ }^{6}$, la emisora de la Universidad Complutense de Madrid y en el perfil de la emisora en Facebook y Twitter.

\section{Mejora de las competencias lingüísticas}

Uno de los resultados más evidentes de la experiencia fue el perfeccionamiento de las competencias lingüísticas en inglés y francés ya que estos dos idiomas fueron, para el 100\% de los participantes, requisito necesario para participar en los programas de radio, en la elaboración de contenidos para las redes sociales y, en general, para la convivencia del grupo. Las competencias en lengua inglesa mejoraron a raíz de la elaboración de los programas de radio cuyos contenidos se emitían en ese idioma, al igual que los contenidos en redes sociales. Por su parte, las competencias y dominio de la lengua francesa fueron adquiridas por los alumnos a lo largo de la convivencia entre los estudiantes franceses y el resto de los miembros de la expedición, así como durante la acogida en domicilios de familias en su estancia en Burdeos.

De igual forma, conviene poner de relieve que, durante las diferentes movilidades, los estudiantes estuvieron en contacto con el idioma del país en las familias de acogida, lo que les permitió un ligero acercamiento a cada una de las lenguas.

\section{Diversidad e interculturalidad como seña de identidad de la Europa de los ciudadanos}

La característica general del grupo estaba basada en la diversidad cultural, religiosa, social, étnica o de razas diferentes; figuraban alumnos musulmanes, cristianos y romanís, entre otros. Dado que el proyecto tenía como objetivo esencial 
el respeto al diferente y pretendía ser una actividad que propiciara la inclusión y el respeto a la diversidad, se incorporaron también en el proyecto a dos alumnos con problemas de movilidad y otro con discapacidad visual. Con esta composición se perseguía luchar contra el fenómeno de la discriminación, la violencia, el acoso y el abandono escolar por bullying. El objetivo fue conseguido ya que ninguno de los componentes tuvo problemas de adaptación y relación con el grupo. Las respuestas de los estudiantes a los cuestionarios pusieron en evidencia que este tipo de experiencia reforzó su formación cultural y su relación con jóvenes de otros países.

Desde el punto de vista de la interculturalidad y la adquisición de competencias culturales, el $100 \%$ de los estudiantes manifestaron que habían ampliado sus conocimientos sobre la realidad social y cultural de la región y el país de acogida en cada una de las movilidades programadas. Las actividades de carácter cultural disfrutadas contribuyeron al conocimiento de la cultura cada país en general y, en particular, de la región que se visitaba. En Salerno (Italia) destacó especialmente la visita al enclave arqueológico romano de Paestrum; en Burdeos (Francia) pudieron conocer de primera mano la cultura vinícola de aquella provincia francesa; durante la estancia de los estudiantes en Madrid asistieron de forma presencial a la producción y emisión en directo de programas en Radio Nacional de España (RNE) y en Telemadrid (RTVM), y ampliaron su conocimiento de España, desde su capital y la ciudad de Toledo. De igual forma, durante la estancia en Brno (República Checa), pudieron adentrarse en la diversidad étnica del país en su visita al Museo de cultura romaní de la ciudad.

\section{Ciudadanía europea}

De las respuestas a las preguntas de los cuestionarios cumplimentados por los alumnos, tanto a la llegada como a la salida de cada país, se deduce que el total de los estudiantes que participaron en el proyecto percibieron que no hay homogeneidad entre los ciudadanos de los países de la Unión Europea. Entre los estudiantes tienen la impresión de que cada país posee sus propias señas de identidad, aunque compartan el espíritu europeo

Existe una opinión unánime entre los estudiantes encuestados al considerar este tipo de movilidad como un instrumento para lograr una mayor conciencia europea. Todos ellos opinaron que este tipo de experiencias sirven de apoyo para la integración de los jóvenes en un proyecto europeo común.

\section{Competencias para un currículum vitae europeo}

Todas las competencias adquiridas por los alumnos a través del proyecto Europa on air fueron acreditadas por cada una de las instituciones educativas en Europass, la plataforma de registro de competencias que permiten al alumno configurar su propio currículum europeo de cualificaciones. Estas credenciales digitales permiten 
a los jóvenes europeos compartir más fácilmente con empresas u oficinas de formación las certificaciones para avalar su participación en el programa Erasmus+ y, por tanto, las competencias adquiridas: culturales, sociales y lingüísticas, entre otras ${ }^{7}$.

Los resultados obtenidos a lo largo de esta experiencia pueden contemplarse tanto desde una perspectiva global, como proyecto transnacional, como desde una óptica local ya que cada país adquirió un protagonismo concreto reflejado en diferentes medios de comunicación. Así, en el caso de España, la experiencia fue recogida, entre otros medios, por la emisora pública Onda Madrid (RTVM), por la revista Tribuna Complutense, en la publicación digital Periodistas.es y en la plataforma digital $i V o o x^{8}$.

\section{DISCUSIÓN/CONCLUSIONES}

Los resultados del proyecto Europa on air confirman las posiciones defendidas por Mar Adalid Donat y Carmen Carmona Rodríguez (2015) que han hecho referencia a las competencias derivadas de la movilidad en relación con la empatía cultural, las habilidades comunicativas y lingüísticas o las actitudes y aptitudes para la movilidad laboral o formativa. No obstante, se pueden extraer cuatro conclusiones generales de esta experiencia.

La primera de ellas pone en valor la contribución de este proyecto a la mejora de las competencias idiomáticas de los estudiantes y a un mayor conocimiento de la Unión Europea, de sus ciudadanos y de su cultura. Algunos autores ya habían puesto de manifiesto que determinadas acciones Erasmus que contemplan cortos espacios de movilidad entre los estudiantes, como es el caso, propician la interculturalidad y la adquisición de competencias lingüísticas entre el profesorado y los estudiantes (Alonso-Marks y Sánchez Hernández, 2020; Nogueras Viceira, 2016). Los resultados de la implementación de este proyecto confirman las posibilidades que ofrecen los contextos multilingües para el aprendizaje de idiomas y para el fomento de la interculturalidad (Alfonzo de Tovar, 2020; Molu et al., 2014). Como en otras experiencias similares, los alumnos pudieron ampliar sus competencias internacionales, especialmente las lingüísticas y las culturales (Molu et al., 2014).

La segunda conclusión justifica la utilización de la educomunicación como método de mejora de las competencias para el trabajo en equipo, para la comunicación interpersonal y para la producción de contenidos formativos. El apoyo a la educación en medios potencia una ciudadanía europea crítica y un maridaje útil entre la educación y la comunicación, en consonancia con posiciones recogidas con anterioridad por la literatura científica (Aguaded, 2013). Además, la experiencia llevada a cabo revela que la webradio y el podcasting son herramientas muy apropiadas y ampliamente aceptadas por los estudiantes para la producción de contenidos educativos y para la comunicación. 
El podcast escolar y universitario es un fenómeno cada vez más popular entre los alumnos, como lo demuestra el hecho de que desde la radio pública y privada se estén propiciando concursos y producciones de contenidos en este formato, lo que pone en valor este proyecto 9 . No obstante, contrasta esta popularidad con estudios de autores que sostienen que queda mucho camino por recorrer para su estudio y ejecución (Galán Arribas et al., 2018; Celaya et al., 2020).

Otra de las conclusiones extraídas del proyecto Europa on air está relacionada con el potencial que ofrecen este tipo de acciones para la interculturalidad. En línea con las posiciones de Pozo-Vicente y Aguaded-Gómez (2012), este programa Erasmus+ no solo fomentó el aprendizaje de la lengua extranjera, sino que además promovió el conocimiento físico de los Estados miembros y la cohesión entre miembros pertenecientes a diferentes culturas, lo que da conciencia de ciudadanía europea entre la población estudiantil. En línea con posiciones mantenidas por Vilá (2006), esta iniciativa Erasmus + se ha consolidado como un instrumento esencial para educar en interculturalidad a estudiantes de distintas edades, orígenes y estratos sociales, utilizando la educación como instrumento para el reconocimiento de la diversidad (Alinder-Ismailova, 2021).

Finalmente, la experiencia de Europa on air consolida la educación no formal como escenario para la educomunicación y la adquisición de competencias relacionadas con los idiomas y la cultura (Iglesias-Vidal, 2014; Alinder-Ismailova, 2021), competencias donde convergen los intereses de los alumnos y la misión de los profesores (Ballesteros Moscosio y Fontecha Blanco, 2019).

\section{NOTAS}

1. Página web oficial de Erasmus plus: https://ec.europa.eu/programmes/erasmusplus/ opportunities/organisations es\#tab-1-2.

2. Página web de Europass: https://europa.eu/europass/es/europass-education-andtraining

3. Página web oficial de Europa on air en español: https://sites.google.com/view/ europeonairspain/

4. Página de Facebook de Europa on Air: https://www.facebook.com/EuropaOnAir

5. Página web oficial de Europa on air en español: https://sites.google.com/site/ europaonair/

6. Programa especial de Europa on air en Inforadio: https://www.ivoox.com/europa-onair sb.html?sb=europa+on+air

7. Página oficial de Europass: https://europa.eu/europass/es/europass-digitalcredentials

8. Programa de Europa on air en Ivoox: http://www.ivoox.com/podcast-programasespeciales sq f1577910 1.html

9. Repositorio de podcast de RTVE: https://www.rtve.es/ buscador?q=Concurso+nacional +podscast 


\section{REFERENCIAS}

Adalid Donat, M., y Carmona Rodríguez, C. (2015). Las competencias interculturales y el programa erasmus. En AIDIPE (Ed.), Investigar con y para la sociedad, $1,(179-$ 188). Cádiz, España: Bubok

Aguaded. I (2013). El programa Media de la Comisión Europea, apoyo internacional a la educación en Medios. Comunicar, $X X(40), \quad 7-8$. https://doi.org/10.3916/ C40-2013-01-01

Aguaded, I., y Martín-Pena, D. (2013). Educomunicación y radios universitarias: panorama internacional y perspectivas futuras. Revista Latinoamericana de Comunicación Chasqui, 124, 63-70. https://doi.org/10.16921/chasqui. voi124.20

Alinder-Ismailova, E. (2021). Socialization and personal competencies in the context of successful intercultural communication. Revista Inclusiones, 8(2), 143-156.

Alfonzo de Tovar, I. (2020). Desarrollo de la competencia intercultural en contextos prurilingües y pluriculturales: programa universitario de movilidad idiomática. Tonos digital: revista de estudios filológicos, 30. http://hdl.handle. net/10201/86463

Alonso-Marks, E., y Sánchez Hernández, E. (2020). El desarrollo intercultural durante estancias cortas en el extranjero: el papel del contacto lingüístico intensivo en la sensibilidad intercultural. Elia. Estudios de lingüística inglesa aplicada, 2o, 13-46 https://doi.org/10.12795/ elia.2020.i20.02

Asenjo Gómez, J. T. (2017). La competencia europeísta: definición del constructo, diseño y validación de un instrumento de medida y análisis de su relación con el programa de movilidad académica Erasmus. Tesis doctoral. Facultad Ciencias Humanas y Sociales. Universidad Pontificia de Comillas.
Asenjo, J. T., y Asenjo Gómez, F. (2020). Educación y europeísmo, un itinerario de encuentro transnacional. Revista Española de educación comparada, 36, 14-31. https://doi.org/10.5944/ reec.36.2020.26145

Ballesteros Moscosio, M. A., y Fontecha Blanco, E. (2019). Competencia Intercultural en Secundaria: Miradas entrelazadas de profesores y estudiantes hacia personas de otras culturas y creencias. Tendencias Pedagógicas, 33, 18-36. https://doi.org/10.15366/ tp2019.33.002

Blas García, J. (2020). Prólogo. En C. MartaLazo, M. A. Ortiz Sobrino, M. Gómez Patiño y J. C. González Pérez (Coord.). COVID-19 y medios de comunicación: voces para un análisis crítico. Sindéresis.

Celaya, I., Ramírez-Montoya, M. S., Naval, C., y Arbués, E. (2020). Usos del podcast para fines educativos. Mapeo sistemático de la literatura en WoS y Scopus (2014-2019). Revista Latina de Comunicación Social, 77, 179-201. https://doi.org/10.4185/ RLCS-2020-1454

Chiappe Laverde, A., y Arias Vallejo, V. (2016). La Educomunicación en entornos digitales: un análisis desde los intercambios de información. Opción, 32(7), 461-479.

Durán Becerra, T., y Tejedor Calvo, S. (2017). Interacción en plataformas educomunicativas. Reflexión sobre tipologías y usabilidad. El Caso de Colombia. Teknokultura. Revista de Cultura Digital y Movimientos Sociales, 14(2), 261-275. https://doi.org/10.5209/ TEKN. 55735

Fallarino, N., Leite Méndez, A., y Cremades, R. (2020). Estudio de caso sobre el desarrollo de la competencia oral en Educación Infantil y Primaria en un centro público. Revista Complutense de 
Educación, 31(3), 319-328. https://doi. org $/ 10.5209 /$ rced.63349

Fuentes Cancell, D. R., Estrada Molina, O., y Delgado Yanes, N. (2021). Las redes sociales digitales: una valoración socioeducativa. Revisión sistemática. Revista Fuentes, 23(1), 41-52. https://doi. org/10.12795/revistafuentes.2021.v23. i1.11947

Galán Arribas, R., Herrero Gutiérrez, F. J., Vergara, M., y Martínez Arcos, C. A. (2018). Estudios sobre el podcast radiofónico: revisión sistemática bibliográfica en WOS y Scopus que denota una escasa producción científica. Revista Latina de Comunicación Social, 73, 1398 - 1411. https://doi.org/10.4185/RLCS2018-1313

García, J. B. (2020). Prólogo. En C. Marta Lazo, M. A. Ortiz Sobrino, M. Gómez Patiño, y J. C. González Pérez, Covid-19 y medios de comunicación: voces para un análisis crítico. Sindéresis.

García Marín, D. (2020). La podcastfera española como comunidad de aprendizaje no formal: Competencias y estrategias educomunicativas desarrolladas por los podcasters independientes. Fonseca. Journal of Communication, 20. https:// doi.org/10.14201/fjc202020201220

González Conde, J., Zamarra-López, M., y Salgado-Santamaría, C. (2018). Radios universitarias: Plurales, abiertas y participativas. Revista Mediterránea de Comunicación/Mediterranean/Journal of Communication, 9(1), 117-135. https:// doi.org/10.14198/MEDCOM2018.9.1.7

Iglesias-Vidal, E. (2014). La formación en interculturalidad en la educación en tiempo libre: necesidades y estrategias desde una perspectiva de competencias interculturales. Revista electrónica interuniversitaria de formación del profesorado, 17(2). https://doi. org/10.6018/reifop.17.2.197511

López Vidales, N., y González Aldea, P. (2014). Audio blogs y TV blogs, herramientas para el aprendizaje colaborativo en Periodismo. Comunicar, 42, 45-53. https://doi.org/10.3916/C422014-04

Martín-Gracia, E., Marta Lazo, C., y GonzálezAldea, P. (2018). La radio universitaria en su dimensión formativa: Estudio de caso de Radio Unizar. Revista Mediterránea de Comunicación / Mediterranean Journal of Communication, 9(1), 49-68. https:// doi.org/10.14198/MEDCOM2018.9.1.3

Molu, E., Başman, M., Eryiğit, D., Tunç, B., y Yaman, G. (2014). Intercultural Education And Incoming-Outcomingof Erasmus Student Study Exchange: Marmara University Students. International Journal for 21 Century Education, 1, 2123. https://www.uco.es/ucopress/ojs/ index.php/ij21ce/article/view/4254

Nogueras Viceira, M. (2016). El programa de movilidad Erasmus y su impacto en la formación intercultural de los futuros docentes de Educación Infantil y Primaria. Trabajo Final de Grado en Maestro de Educación Primaria Mención de Lengua Extranjera Inglés. Universidad de Granada.

Ortiz Sobrino, M. A., Rodríguez Barba, D., y Jacques Cheval, J. (2014). La radio universitaria en el siglo XXI: perspectiva hispano-francesa. EDMETIC, Revista de Educación Mediática y TIC, 3(1), 6186. https://doi.org/10.21071/edmetic. v3i1.2881

Ortiz-Sobrino, M. A., Gómez López, J., MartaLazo, C., y Peñalva-Tobías, S. (2020). Revisión de la literatura científica de la radio universitaria como instrumento de educación mediática. CIC. Cuadernos de Información y Comunicación, 25, 257268. https://doi.org/10.5209/ciyc.68277

Pérez-Femenía, E., e Iglesias-García, M. (2020). La radio escolar como mediadora en el aprendizaje del uso del móvil en la adolescencia. Zer, 25(48), 329-345. https://doi.org/10.1387/zer.21583 
Pozo-Vicente, C., y Aguaded-Gómez, J. I. (2012). El programa de movilidad ERASMUS: motor de la adquisición de competencias interculturales. Revista de Investigación Educativa, 3o(2), 441-458. https://doi.org/10.6018/rie.30.2.138191

Rendón López, L., y Ortega Carrillo, J. A. (2015).Aprendizaje colaborativo mediante redes sociales y radio comunitaria web 2.0. Revista Lasallista de investigación, 12(2). 54-65. https://doi.org/10.22507/ rli.v12n2a6

Rodríguez García, Y. M. (2017). Reconceptualization of education in the digital era: educommunication. Revista de Comunicación de la SEECI, XXI(42), 85-118. $\quad$ https://doi.org/10.15198/ seeci.2017.42.85-118

Tur, G., Urbina, S., y Buchem, I. (2019). El proyecto E+ Open Virtual Mobility para el Espacio Europeo de Educación Superior. En R. Roig-Vila (Coord.), Redes de Investigación e Innovación en Docencia Universitaria. Volumen 2019 (pp. 427436). Universidad de Alicante/Instituto de Ciencias de la educación.

Vázquez, S. (2007). TIC y podcast. Padres y Maestros / Journal of Parents and Teachers, 311, 36-39. https:// revistas.comillas.edu/index.php/ padresymaestros/article/view/14325
Vilá, R. (2006). La dimensión afectiva de la competencia comunicativa intercultural en la Educación Secundaria Obligatoria: Escala de Sensibilidad Intercultural. Revista de Investigación Educativa, 24(2), 353-372.

\section{Webgrafía}

Comisión Europea (2021). Programmes Erasmus-plus - Opportunities. https:// ec.europa.eu/programmes/erasmusplus/ opportunities/organisations es\#tab-1-2

Comisión Europea (2021). Website of the European Union. https://ec.europa. eu/programmes/erasmus-plus/book/ export/html/300 es

Europass / Unión Europea (2021). https:// europa.eu/europass/es/europasseducation-and-training

Europa on air / España (2018). https://sites. google.com/site/europaonair/

iVoox (2021). Podcast. http://www.ivoox. com/podcast-programas-especiales sq $\underline{\text { f1577910 1.html }}$

iVoox (2021). Podcast. https:// www.ivoox.com/europa-on-air sb.html?sb=europa+on+air

RNE: Concurso nacional de podcast. https:// www.rtve.es/buscador?q=Concurso+nacional+podscast

\section{Redes Sociales}

Facebook. Perfil Europa on air. https://www.facebook.com/EuropaOnAir Instagram. Perfil Europa on air. https://www.instagram.com/europaonair/

\section{PERFIL ACADÉMICO Y PROFESIONAL DE LOS AUTORES}

Miguel Ángel Ortiz Sobrino. Profesor Titular de la Universidad Complutense de Madrid. Director del Master de Realización y Diseño de programas y formatos en televisión. Investigador en el proyecto "Youtubers e instagrammers: la competencia mediática en los prosumidores emergentes”. MEC. RTI2018-093303-B-Ioo.I.P. https://orcid.org/0000-0003-0103-9524 


\section{DIRECCIÓN DEL AUTOR}

Universidad Complutense de Madrid (UCM).

Facultad de Ciencias de la Información

Avenida Complutense s/n.

28040 Madrid (España)

Nereida López Vidales. Profesora Titular de Periodismo de la Universidad de Valladolid. Es Doctora en Ciencias Políticas y Sociología, periodista, socióloga, politóloga y Máster en Producción Radiofónica. Directora del Observatorio del ocio y entretenimiento digital (GIR OCENDI)-Directora de Radio UVA. Coordinadora del Grado en Periodismo y el Programa de Doctorado en la Universidad de Valladolid. https://orcid.org/0000-0002-6960-6129

\section{DIRECCIÓN DE LA AUTORA}

Universidad de Valladolid

Dpto. Historia Moderna, Contemporánea y de América.

Periodismo, Comunicación Audiovisual y Publicidad

Facultad de Filosofía y Letras

Plaza del Campus universitario s/n

47011 Valladolid (España)

Juan Carlos González Pérez. Periodista. Doctorando en el Programa de doctorado de Periodismo de la Universidad Complutense de Madrid. PDI colaborador en la Universidad de Educación a Distancia. https://orcid.org/o0oo-0002-66449682

\section{DIRECCIÓN DEL AUTOR}

Universidad Complutense de Madrid (UCM).

Facultad de Ciencias de la Información

Avenida Complutense s/n.

28040 Madrid (España)

Fecha de recepción del artículo: 12/04/2021

Fecha de aceptación del artículo: 05/06/2021

Fecha de aprobación para maquetación: 03/08/2021 